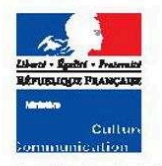

Secrétariat général

Service de la
coordination des

politiques culturelles

et de l'innovation

Département

des études,

de la prospective
et des statistiques

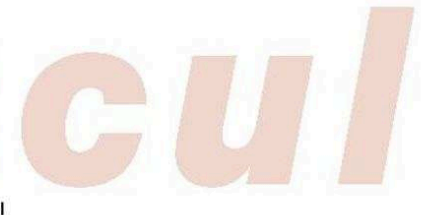

182, rue Saint-Honoré, 75033 Paris cedex 0

풍 0140157917 - 国 0140157999

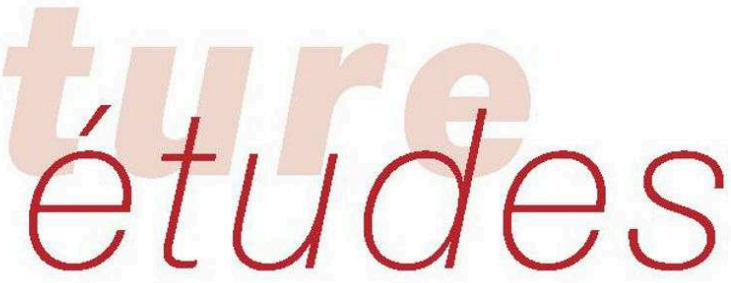

\title{
Politiques d'éducation artistique et culturelle : rôle et action des collectivités locales
}

Françoise ENEL*

Artistic and cultural education policies:
the role and action of local authorities

\section{Avant-propos}

Vingt ans de politique d'éducation artistique et culturelle initiée par l'État par le bras des ministères chargés de la Culture et de l'Éducation nationale et leurs services déconcentrés (Drac et rectorat) ont essaimé sur l'ensemble du territoire une multitude de dispositifs, diversement appropriés et mis en ouvre par les collectivités territoriales.

L'implication croissante des acteurs locaux - institutions culturelles et associations socioculturelles, enseignants, artistes - et l'engagement des communes et des intercommunalités, soutenus par les départements et les régions pour construire des dispositifs cohérents au service de l'éducation artistique de l'enfant, méritaient une analyse précise. Si la diversité des configurations locales ne peut se réduire à quelques catégories et illustre la variêté des dynamiques locales à l'ouvre, cinq grands types peuvent néanmoins être dégagés suivant des carac téristiques communes (dispositifs et structuration des relations entre institutions et acteurs locaux), qui permettent de structurer l'analyse.

Sans être évaluative, cette esquisse de typologie aide à penser l'action publique en matière d'éducation artistique et culturelle, dans un contexte de redéfinition des politiques culturelles de l'État et des dif férents échelons territoriaux, sous l'impulsion de mutations numériques et de repositionnement du rôle et de l'action des différents acteurs de la sphère publique.

P. C.
L'éducation artistique et culturelle vise à former chez les enfants et les adolescents la capacité à poser un regard personnel sur le monde, grâce à la connaissance des cuvres et des courants artistiques qui ont marqué l' histoire de l'humanité. Sollicitant la sensibilité des enfants, les actions en ce domaine s'organisent autour de trois axes majeurs : le rapport direct aux ceuvres, l'approche analytique et cognitive des œuvres, et enfin la pratique effective dans le cadre d'ateliers.

Le développement des initiatives prises en la matière par les collectivités territoriales depuis une vingtaine d'années, qui vont bien au-delà des obligations légales, et un déficit de connaissances de l'ensemble des interventions publiques ont conduit le ministère de la Culture et de la Communication à identifier l'ensemble des types d'interventions à partir d'études de cas. Leur analyse permet de dégager une première classification et de saisir l'articulation de deux enjeux majeurs dans ce domaine, celui de la refondation du partenariat entre l'État et les collectivités territoriales, ainsi que l'enjeu de la structuration de politiques éducatives et culturelles territoriales : quelles conditions de passage de la juxtaposition de dispositifs à l'avènement de véritables politiques territoriales, quelles stratégies, quels leviers ?

L'attention portée à la structuration de la politique d'éducation artistique et culturelle au niveau territorial et aux stratégies déployées ou à développer privilégie trois angles de vue :

- une mise en perspective historique qui permet de saisir l'évolution des relations entre l'État et les collectivités territoriales

- une analyse du jeu des acteurs et des stratégies développées dans les territoires étudiés ;

- la mise en exergue de défis d'avenir relatifs au sens et aux modalités des politiques d'éducation artistique et culturelle.

L'état des lieux, élaboré à partir de l'analyse de six sites dans deux régions (Nord - Pas-de-Calais et Pays de la Loire), constitue une première étape ver une meilleure appréhension de la richesse des politiques locales d'éducation artistique et culturelle et des actions conduites sur les territoires. Limité à deux régions, il ne prétend pas épuiser la diversité des situations locales. Cette

* Vérès Consultants. Étude réalisée avec la collaboration de Cécile Delesalle, Cyrille GaZave et Sophie Govindassamy. 


\title{
Politiques d'éducation artistique et culturelle : rôle et action des collectivités locales
}

\author{
Artistic and cultural education policies: the role and action of local authorities
}

Enel Françoise

Éditeur : Département des études, de la prospective et des statistiques

Lieu d'édition : Paris

Année d'édition : 2011

Date de mise en ligne : 8 juillet 2015

Collection : Culture études

ISBN électronique : 9782111398962

\section{Sbooks}

http://books.openedition.org

\section{Édition imprimée}

Date de publication : 1 avril 2011

Nombre de pages : 8

\section{Référence électronique}

FRANÇOISE, Enel. Politiques d'éducation artistique et culturelle : rôle et action des collectivités locales.

Nouvelle édition [en ligne]. Paris : Département des études, de la prospective et des statistiques, 2011 (généré le 25 avril 2021). Disponible sur Internet : <http://books.openedition.org/deps/188>. ISBN : 9782111398962. 

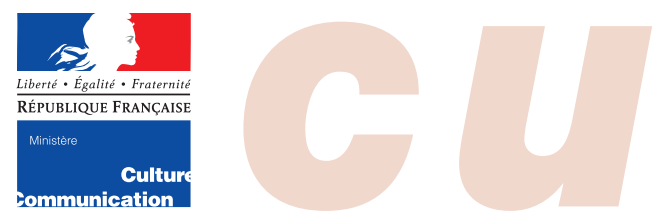

Secrétariat général

Service de la

coordination des

politiques culturelles

et de l'innovation

Département

des études,

de la prospective

et des statistiques

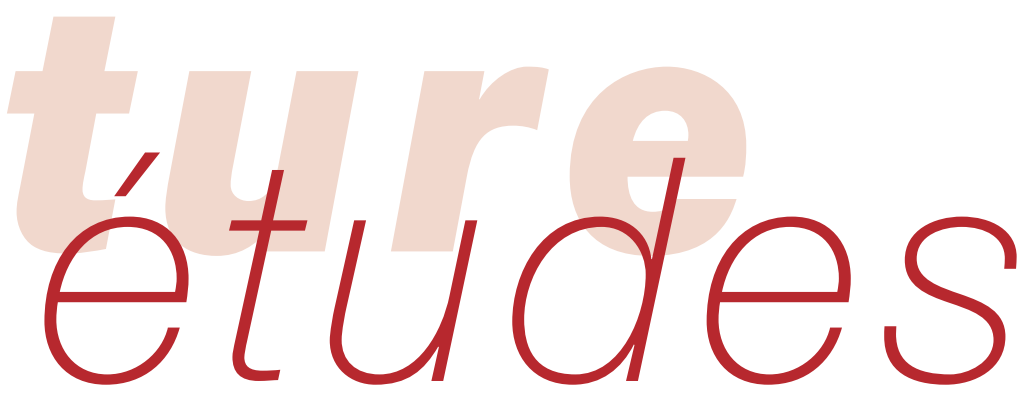

\section{Politiques d'éducation artistique et culturelle : rôle et action des collectivités locales}

\section{Françoise ENEL ${ }^{*}$}

\author{
Artistic and cultural education policies: \\ the role and action of local authorities
}

\section{Avant-propos}

Vingt ans de politique d'éducation artistique et culturelle initiée par l'État par le bras des ministères chargés de la Culture et de l'Éducation nationale et leurs services déconcentrés (Drac et rectorat) ont essaimé sur l'ensemble du territoire une multitude de dispositifs, diversement appropriés et mis en ouvre par les collectivités territoriales.

L'implication croissante des acteurs locaux - institutions culturelles et associations socioculturelles, enseignants, artistes - et l'engagement des communes et des intercommunalités, soutenus par les départements et les régions pour construire des dispositifs cohérents au service de l'éducation artistique de l'enfant, méritaient une analyse précise. Si la diversité des configurations locales ne peut se réduire à quelques catégories et illustre la variété des dynamiques locales à l'ouvre, cinq grands types peuvent néanmoins être dégagés suivant des caractéristiques communes (dispositifs et structuration des relations entre institutions et acteurs locaux), qui permettent de structurer l'analyse.

Sans être évaluative, cette esquisse de typologie aide à penser l'action publique en matière d'éducation artistique et culturelle, dans un contexte de redéfinition des politiques culturelles de l'État et des différents échelons territoriaux, sous l'impulsion de mutations numériques et de repositionnement du rôle et de l'action des différents acteurs de la sphère publique.

P. C.
L'éducation artistique et culturelle vise à former chez les enfants et les adolescents la capacité à poser un regard personnel sur le monde, grâce à la connaissance des œuvres et des courants artistiques qui ont marqué l'histoire de l'humanité. Sollicitant la sensibilité des enfants, les actions en ce domaine s'organisent autour de trois axes majeurs : le rapport direct aux œuvres, l'approche analytique et cognitive des œuvres, et enfin la pratique effective dans le cadre d'ateliers.

Le développement des initiatives prises en la matière par les collectivités territoriales depuis une vingtaine d'années, qui vont bien au-delà des obligations légales, et un déficit de connaissances de l'ensemble des interventions publiques ont conduit le ministère de la Culture et de la Communication à identifier l'ensemble des types d'interventions à partir d'études de cas. Leur analyse permet de dégager une première classification et de saisir l'articulation de deux enjeux majeurs dans ce domaine, celui de la refondation du partenariat entre l'État et les collectivités territoriales, ainsi que l'enjeu de la structuration de politiques éducatives et culturelles territoriales : quelles conditions de passage de la juxtaposition de dispositifs à l'avènement de véritables politiques territoriales, quelles stratégies, quels leviers?

L'attention portée à la structuration de la politique d'éducation artistique et culturelle au niveau territorial et aux stratégies déployées ou à développer privilégie trois angles de vue :

- une mise en perspective historique qui permet de saisir l'évolution des relations entre l'État et les collectivités territoriales ;

- une analyse du jeu des acteurs et des stratégies développées dans les territoires étudiés :

- la mise en exergue de défis d'avenir relatifs au sens et aux modalités des politiques d'éducation artistique et culturelle.

L'état des lieux, élaboré à partir de l'analyse de six sites dans deux régions (Nord - Pas-de-Calais et Pays de la Loire), constitue une première étape vers une meilleure appréhension de la richesse des politiques locales d'éducation artistique et culturelle et des actions conduites sur les territoires. Limité à deux régions, il ne prétend pas épuiser la diversité des situations locales. Cette

\footnotetext{
* Vérès Consultants. Étude réalisée avec la collaboration de Cécile Delesalle, Cyrille GaZave et Sophie Govindassamy.
} 
exploration illustre la vitalité de l'éducation artistique et culturelle dans les collectivités et la diversification croissante des stratégies déployées, selon les enjeux poursuivis localement et les configurations d'acteurs en présence.

\section{UNE NOUVELLE ÉTAPE DE DÉVELOPPEMENT DE L'ÉDUCATION ARTISTIQUE ET CULTURELLE}

L'intérêt pour l'éducation artistique n'est pas nouveau et l'intérêt des acteurs, tant institutionnels que culturels, pour ce domaine s'est renforcé au cours des deux dernières décennies, au point que celui-ci a pris une place d'importance dans l'action publique culturelle. Les finalités poursuivies ont été réaffirmées au travers de textes programmatiques; des stratégies élaborées ont été définies et des réseaux d'acteurs plus nombreux ont contribué à leur mise en œuvre dans les territoires.

\section{Un développement initialement porté par l'État}

Impulsée par l'État, l'éducation artistique et culturelle s'est historiquement appuyée sur la double action des ministères chargés de la Culture et de l'Éducation nationale et de leur représentation régionale : Drac et rectorat. Elle a mobilisé les enseignants et concerné prioritairement les enfants dans le cadre scolaire pour des raisons à la fois philosophiques (égalité de l'accès à l'éducation), pratiques (cadre stable et familier pour l'enfant) et politiques, afin de permettre ainsi l'atteinte de l'objectif de démocratisation. La diffusion de la politique d'éducation artistique et culturelle s'est faite au moyen de divers dispositifs : classes du patrimoine, ateliers de pratique artistique, classes à parcours artistique et culturel..., relayés par les conseils régionaux en direction des lycéens et les conseils généraux en direction des collégiens, par l'intermédiaire de programmes comme Collège au cinéma ou au théâtre, Lycéens et apprentis au cinéma, etc., auxquels les collectivités locales ont souscrit et apporté leur soutien.

Trois mouvements ont caractérisé cette phase de développement et de consolidation de l'éducation artistique et culturelle au cours des dix dernières années. Le premier concerne l'accroissement et la diversification des propositions, rendus possibles par l'augmentation des financements publics. Cette explosion de l'offre s'est accompagnée de transformations dans la forme et les contenus, en raison de la perméabilité de l'action culturelle aux grands courants qui traversent la culture, comme le développement de la pluridisciplinarité dans la création artistique.

Le deuxième mouvement renvoie à l'amélioration de toute la chaîne d'élaboration des propositions d'éducation artistique et culturelle. La qualification progressive de l'offre a emprunté des voies diverses, allant de la formulation de règles et de prérequis qui se sont affinés au fil du temps (comme la nécessité d'un travail en classe en amont et en aval de la proposition d'éducation artistique et culturelle ou l'articulation du projet avec les autres apprentissages scolaires...), de l'amélioration de l'accueil et des espaces dédiés, au développement progressif de procédures de suivi et d'évaluation des actions... Ces évolutions traduisent une même volonté des acteurs de donner du sens et de la cohérence à l'éducation artistique et culturelle par un travail inscrit dans la durée, capable d'éveiller la curiosité des enfants grâce à un croisement des regards, des disciplines et des modes d'intervention.
Parallèlement, la volonté d'une qualification des acteurs a favorisé des politiques visant à la clarification des missions et des compétences recherchées (spécification du rôle et de la place de l'artiste, des médiateurs et des coordonnateurs, par exemple), l'amélioration de la sélection des opérateurs, la mise en place d'un accompagnement adapté et d'un accès à la formation. L'offre de formation s'est développée, diversifiée dans ses contenus, en direction des publics (enseignants, chefs d'établissement, opérateurs culturels, personnels communaux), mais aussi dans ses modalités de réalisation, notamment par le développement de formations croisées (Délégation académique à l'action culturelle, associations et équipements culturels).

Le troisième mouvement observable au cours des deux dernières décennies concerne l'élargissement du système d'acteurs, l'approfondissement des partenariats (entre institutions, entre institutions et structures culturelles, entre structures culturelles, entre établissements scolaires...) et le développement des coopérations sous les impulsions conjointes des institutions aux différents échelons territoriaux, des opportunités (matérielles ou stratégiques) et, plus récemment, de dynamiques de mutualisation visant à optimiser l'élaboration et la diffusion de l'offre ou à faire face à la baisse des moyens.

L'ensemble des évolutions analysées précédemment, qu'elles touchent à l'organisation et aux fonctionnements, à une plus grande compétence des acteurs par la formation, à l'amélioration de leur information et à la diversification des modalités d'accompagnement, ont contribué à renforcer la qualification des propositions d'éducation artistique et culturelle et à les rendre visibles de l'ensemble des acteurs. Elles participent de ce mécanisme global par lequel l'éducation artistique et culturelle est devenue un «objet social», jugé consensuel aujourd'hui.

\section{Une implication croissante des collectivités locales}

Le processus de consolidation de l'éducation artistique et culturelle, qui a inscrit la préoccupation d'éducation artistique et culturelle au cœur des structures (établissements scolaires et équipements culturels), a peu concerné, sauf exception, les collectivités territoriales. Dans leur majorité, celles-ci semblent avoir bougé davantage sous l'influence d'incitations externes - en lien avec l'action des acteurs publics des autres échelons territoriaux et de grandes structures culturelles - que d'une réflexion interne sur l'optimisation des politiques locales d'éducation artistique et culturelle. Cette réflexion est actuellement en cours, sous l'effet conjugué du transfert de charges croissant vers les collectivités territoriales, de la répartition des compétences culturelles entre communes et intercommunalités et du développement quantitatif de l'offre sur la période, qui contribue à soumettre l'éducation artistique et culturelle à de nouveaux enjeux.

Initiées par l'État, ces politiques ont été réappropriées, à des rythmes différents selon les configurations locales, tant par les structures culturelles que par des collectivités qui ont inscrit l'action culturelle à leur programme et ont cherché à se doter d'équipements et de compétences idoines. L'intégration de la mission éducative dans l'organisation du service culturel local, des équipements ou des structures s'est faite de manière variable selon les sites, à des rythmes et avec des moyens très différents. Le poids des cultures et des situations politiques locales, les logiques de découpage interne des services et les modes d'inscription de la compétence culturelle dans les organigrammes expliquent qu'il n'existe pas de schéma d'organisation dominant qui se serait imposé progressivement aux collectivités. 


\section{Vers un nouveau modèle de développement}

Après une phase de croissance où chacun (ou presque) a pu trouver sa place en se juxtaposant aux autres, sans que le manque de schéma d'ensemble ne soit préjudiciable, on atteint aujourd'hui une nouvelle étape, marquée par le repositionnement des acteurs, avec le passage d'une approche «territorialisée » de l'éducation artistique et culturelle (fortement impulsée par l'État dans le cadre de politiques contractuelles autour de dispositifs définis par l'administration centrale) à une approche «territoriale » (c'est-à-dire portée et largement financée par les acteurs locaux et cherchant à prendre en compte les spécificités sociopolitiques des territoires), et par une volonté de rationalisation de l'offre d'éducation artistique et culturelle. L'implication des collectivités territoriales, la nécessité d'optimiser des financements en baisse, le manque de personnel des équipes régionales et départementales, l'essoufflement du système du fait de l'incapacité de certaines structures à développer leur offre au regard de leurs moyens ou de réticences des enseignants en raison de contraintes accrues, obligent à concevoir un nouveau schéma organisationnel et d'autres modes de gouvernance. Après une période durant laquelle les usages et les hiérarchies en place ont été implicitement préservés, qu'il s'agisse des hiérarchies entre acteurs institutionnels ou entre structures culturelles, dans la mesure où l'impulsion venait prioritairement du sommet, l'apparition de nouveaux décideurs issus de l'échelon territorial modifie la règle du jeu. On assiste à une « localisation » de l'organisation qui concerne aussi l'éducation artistique et culturelle, et qui s'appuie d'une part sur les collectivités en capacité de porter une politique d'éducation artistique et culturelle et, d'autre part, la formation d'un réseau de collectivités publiques soucieuses de mettre en place une politique en direction des territoires autour de la culture.

Cette montée en puissance des territoires s'enracine dans la conjonction de deux évolutions, qui amplifient la dynamique en cours : la première concerne le niveau de maturité atteint par des communes ou des intercommunalités dont l'implication fonctionnelle et financière va croissant, et dont les politiques publiques ont permis de qualifier les pratiques; la seconde renvoie au fait que l'éducation artistique et culturelle est souvent perçue comme un levier pertinent d'une politique territoriale en raison de sa forte légitimité et de sa nature transversale.

La mobilisation des élus locaux s'inscrit également dans une logique de rééquilibrage territorial et dans une logique de rationalisation de la politique et des ressources. Il s'agit, par une action mieux coordonnée entre les différents échelons territoriaux, d'aider les communes à structurer un projet de territoire, et de leur permettre d'anticiper la réforme territoriale de 2012. Il s'agit, en outre, par le soutien aux projets locaux, d'aider les intercommunalités à développer un projet culturel, à promouvoir des démarches de mutualisation des ressources entre communes et à poursuivre le mouvement de qualification de l'offre. Cette politique présente l'avantage de permettre aux élus de recourir à de nouveaux acteurs, jugés plus professionnels. Aux niveaux communal et intercommunal, il s'agit, selon des responsables locaux, de promouvoir « un service public partagé », en cherchant à faire travailler ensemble et à coordonner la totalité des acteurs qui contribuent collectivement à l'action culturelle sur un territoire.

Cette nouvelle donne représente plus qu'un simple ajustement des politiques d'éducation artistique et culturelle. Questionnant la division du travail entre les différents niveaux territoriaux, la segmentation des compétences héritées du passé : des communes en charge du primaire, un conseil général en charge des collèges et un conseil régional en charge des lycées, elle est porteuse de véritables changements. Elle interroge la place de l'Éducation nationale dans ce schéma de politique territoriale d'éducation artistique et culturelle, et les leviers de mobilisation des communes ou des intercommunalités encore peu sensibilisées ou qui craignent de s'engager dans un processus dispendieux au regard des finances locales, alors que la crise économique oblige à redéfinir les priorités de politique locale.

Cette «politique des territoires » s'accompagne d'une transformation des méthodologies d'action des acteurs publics historiques : désignation de territoires prioritaires d'intervention, désignation de nouveaux relais, qu'il s'agisse de représentants de collectivités ou de structures culturelles têtes de réseau qui font travailler ensemble les divers partenaires (collectivités, enseignants, artistes et opérateurs culturels), la signature de contrats de territoire, qui impliquent des méthodologies de diagnostic et d'intervention partagées. Elle s'accompagne d'une diversification des modes d'investissement et d'intervention des collectivités locales dans l'éducation artistique et culturelle, qui rend plus complexe, en retour, l'intervention des autres échelons territoriaux, régionaux et départementaux, obligés de composer avec les priorités et les logiques d'action de ces acteurs.

\section{Cing SCHÉMAS LOCAUX D'ORGANISATION DE L'ÉDUCATION ARTISTIQUE ET CULTURELLE}

Dans le secteur de l'éducation artistique et culturelle comme dans d'autres domaines publics, le pilotage national n'a pas conduit à une uniformisation territoriale des politiques et des organisations, pour des raisons qui tiennent à la diversité des réalités sociopolitiques régionales et à la plus ou moins grande mobilisation des acteurs locaux et des équipements culturels.

Trois types de territoires peuvent être distingués, selon l'origine de l'impulsion de la politique d'éducation artistique et culturelle mise en place: interne, c'est-à-dire portée prioritairement par la commune ou l'intercommunalité ; portée un tandem institution territoriale départementale ou régionale et commune ou intercommunalité ou institution scolaire et acteurs culturels; ou encore relevant de l'intervention d'acteurs extérieurs au territoire, qu'il s'agisse d'acteurs institutionnels, par l'intermédiaire des dispositifs spécifiques, ou d'opérateurs culturels.

L'analyse des situations étudiées permet de dégager cinq configurations locales de mise en œuvre de l'éducation artistique et culturelle (qui ne sont pas présentées ici dans un ordre décroissant de légitimité ${ }^{1}$ :

- une politique intégrée, tout au moins dans son projet, même s’il peut exister des écarts entre le projet et sa réalisation ;

- une approche ciblée autour d'axes stratégiques identifiés ;

- une gouvernance multipolaire, animée par des acteurs de statuts différents ;

- une approche segmentée, caractérisée par la juxtaposition d'un certain nombre d'actions et de dispositifs portés par des acteurs différents, qui peuvent se trouver en situation de concurrence ;

1. La taille de l'échantillon ne prétend pas épuiser la diversité des configurations locales possibles ni proposer des organisations et des fonctionnements archétypiques. 
- une situation où l'impulsion est externe, dans des territoires où la sensibilisation à l'éducation artistique et culturelle est rythmée par la participation à des projets initialisés par des acteurs institutionnels ou associatifs extérieurs à la zone (même si des offres internes existent, parfois de longue date, mais qui sont des offres d'équipements et non des offres de territoire).

\section{Une politique intégrée d'éducation artistique et culturelle}

Cette stratégie ambitieuse se retrouve dans des communes de taille variable mais qui ont en commun de bénéficier d'élus et de personnels sensibilisés à la culture, de consacrer des moyens importants à son développement et de placer l'éducation artistique et culturelle au cœur de la politique culturelle.

Elle se caractérise par une volonté affirmée d'articuler au nom d'un principe de cohérence :

- les différents services communaux concernés, à un titre ou à un autre, par la mise en œuvre de l'éducation artistique et culturelle;

- les politiques éducative, culturelle, de la jeunesse, d'éducation artistique et culturelle, voire de rénovation urbaine dans leur volet de mobilisation de la population par des projets à composante culturelle ;

- les divers dispositifs d'éducation artistique et culturelle, qu'ils soient régionaux, départementaux ou locaux ;

- les quartiers de la commune, dans un souci de rééquilibrage territorial et de lutte contre la relégation de certains d'entre eux ; - les différents champs disciplinaires de la création artistique ;

- les temps de l'enfant, avec la préoccupation de promouvoir des parcours d'éducation culturelle et artistique tout au long du cursus scolaire (voire au-delà) et cherchant à relier temps scolaire et hors temps scolaire.

La mise en œuvre de cette politique intégrée a pu conduire à l'implantation d'une armature d'équipements culturels de proximité et à la création de nouveaux équipements transversaux (du type maison des arts). Elle a contribué à consolider la place de la direction des affaires culturelles (quel que soit l'intitulé exact de la direction), chargée de centraliser les politiques culturelles, d'éducation artistique et culturelle et de relation aux publics.

Au plan stratégique, elle s'accompagne d'une volonté de mise en cohérence des offres d'éducation artistique et culturelle que celles-ci s'inscrivent dans la politique culturelle ou éducative locale, dans le programme de réussite éducative, dans la politique de la ville ou dans la politique jeunesse. Ainsi l'une des communes étudiées a fait du projet éducatif local (PEL) l'instrument de l'intégration et de la coordination des différentes propositions d'éducation artistique et culturelle, ce qui lui permet d'améliorer la visibilité de l'offre à l'échelle de la ville et d'ajuster les propositions aux besoins identifiés localement. Ces collectivités, tout en cherchant à ne pas s'adresser aux enfants uniquement dans le cadre scolaire, apparaissent par ailleurs soucieuses d'un partenariat dynamique avec l'Éducation nationale et ses représentants locaux, qui va bien au-delà des actions en faveur de leur public de compétence que sont les enfants du primaire.

$\mathrm{Au}$ plan des ressources humaines, ces communes mènent des politiques proactives de formation des personnels municipaux, voire des associations culturelles locales, afin de les ouvrir à la création artistique professionnelle et d'améliorer leurs compétences dans la transmission culturelle.

Si la dimension intercommunale ou d'agglomération n'est pas absente dans cette configuration, elle est portée essentiellement par des structures ou des associations culturelles à fort rayonnement géographique plus que par la ville, ou encore elle s'appuie sur des liens privilégiés et choisis avec d'autres communes (en fonction d'affinités personnelles d'élus ou de techniciens) mais en s'affranchissant du cadre administratif de l'intercommunalité, une intercommunalité de facto en quelque sorte. En ce sens, ce mode de fonctionnement présente des limites: risque d'un repli possible sur le territoire de la commune, faible participation à des politiques intercommunales, lorsqu' elles existent, et tendance à voir dans ces dernières une menace pour le système élaboré localement (crainte de la perte de liberté d'action des acteurs communaux et d'une baisse de qualité des propositions d'éducation artistique et culturelle...).

\section{Une politique ciblée d'éducation artistique et culturelle}

Cette politique ne poursuit pas le même objectif globalisant que la configuration précédente, mais elle est fondée sur la volonté de définir des priorités d'action à partir desquelles organiser l'éducation artistique et culturelle à l'intérieur d'un même périmètre ou d'un regroupement de communes.

Les études de terrain ont permis d'identifier plusieurs stratégies dans le cadre de cette approche ciblée de l'éducation artistique et culturelle. La définition de pôles d'intervention représente l'une d'elles et s'inscrit soit dans une logique d'excellence en fléchant les moyens sur des champs artistiques ou des technologies précises, de manière à assurer un savoir-faire et une notoriété dans le ou les domaines considérés, donnant ainsi une visibilité de la collectivité à l'extérieur, soit dans une logique de spécialisation: plusieurs communes associées décident de s'investir prioritairement dans telle ou telle discipline artistique et de créer des synergies entre territoires, afin de limiter le saupoudrage des moyens et de proposer des offres de qualité. Ces pôles peuvent se décliner à différentes échelles territoriales, depuis la commune, l'intercommunalité, des associations volontaires de communes à des échelles plus vastes de bassin de vie ou de région.

Les autres stratégies apparaissent plus tactiques. Les unes s'inscrivent dans une volonté de procéder par étapes : les acteurs privilégient une démarche progressive et définissent des priorités d'action (la lecture, par exemple, ou le spectacle vivant autour d'un festival jeune public) soit parce qu'ils n'ont pas les moyens d'aborder tous les secteurs, soit encore parce qu'ils cherchent à consolider le système d'acteurs autour d'un ou de deux domaines privilégiés, à l'occasion du transfert partiel ou intégral de la compétence culturelle à l'intercommunalité par exemple, avant de s'intéresser à des champs plus complexes (comme celui de la musique...). Les autres s'inscrivent dans une logique pragmatique, fondée sur la prise en compte des rapports de force locaux et le respect des hiérarchies en place : l'éducation artistique et culturelle est menée avec les acteurs institutionnels et culturels investis dans le champ. Il s'ensuit une faiblesse des liens avec d'autres dispositifs à composante culturelle et avec d'autres acteurs positionnés sur l'action socioculturelle ou les politiques en direction de la jeunesse.

\section{Une gouvernance multipolaire}

Dans cette configuration, des acteurs importants mais de statuts différents organisent et animent l'éducation artistique et culturelle sur le territoire, avec l'aide financière du département et de la région. Dans ce cas, le pilotage de l'éducation artistique et culturelle s'opère à la fois : 
- par des communes très investies, qui sont sources de propositions multiples, à côté de communes peu mobilisées ;

- par des structures culturelles qui ne se cantonnent pas à un rôle d'opérateurs en élaborant des propositions mais contribuent activement à la mobilisation des communes et à la formation des acteurs (enseignants, autres opérateurs culturels...) ;

- par des établissements d'enseignement artistique qui jouent un rôle structurant en intervenant auprès de petits établissements et d'associations pour les former et les faire travailler en réseau.

Ce fonctionnement multipolaire apparaît performant du point de vue de la diversité et de la qualité de l'offre d'éducation artistique et culturelle, en raison de l'émulation qui se crée entre les protagonistes et du fait que les différents acteurs sont dotés de suffisamment de légitimité et de pouvoir pour peser, directement ou indirectement, sur tout ou partie du système. Sa limite procède de la complexification et du niveau d'aboutissement de la coordination des acteurs et des projets, variable selon les cas. De plus, malgré leur légitimité reconnue, tous les acteurs ne sont pas égaux et certains d'entre eux - les structures culturelles notamment - sont confrontés à une fragilité structurelle liée à la baisse possible de leurs financements dans un contexte de restriction budgétaire.

\section{Une approche segmentée}

Cette configuration ne signifie pas une absence d'offres culturelles et d'éducation artistique et culturelle de qualité sur le territoire, mais celles-ci proviennent de niveaux territoriaux et d'opérateurs différents (médiateurs communaux, structures culturelles, parc naturel régional, opérateurs privés...), qui s'ignorent ou se vivent en concurrence. Les actions se juxtaposent les unes aux autres, en l'absence de lieux de coordination de la politique d'éducation artistique et culturelle, et se régulent via une logique de l'offre et de la demande, les enseignants en bout de chaîne étant preneurs, ou non, de tel ou tel projet qui leur est proposé.

Dans un contexte de manque de lisibilité de l'offre, des tentatives de mise en cohérence existent, soutenues par différentes catégories d'acteurs (département, pays, intercommunalité) mais souvent limitées à des sous-ensembles territoriaux ou thématiques ou à des sous-ensembles d'acteurs (bassin d'éducation, par exemple). De même, les velléités de travail en réseau de certains acteurs s'affranchissent des périmètres institutionnels et ces réseaux, fondés sur des logiques affinitaires, viennent s'ajouter aux autres circuits en place.

Dans ce schéma, les rivalités potentielles entre acteurs ou entre territoires rendent difficile l'émergence d'un acteur interne en capacité de réguler le système. Celui-ci doit vraisemblablement être stimulé et accompagné, dans un premier temps, par un acteur externe (ou un binôme) capable de rassembler autour d'une même table les divers acteurs de l'éducation artistique et culturelle localement.

\section{Un territoire travaillé de l'extérieur}

Ce mode de fonctionnement caractérise principalement des territoires ruraux excentrés. Bien qu'à l'écart des hauts lieux de la vie culturelle, ils ne sont pas dépourvus d'équipements culturels (de type écomusée, par exemple) ni d'associations culturelles souvent très actives. L'histoire récente révèle que ces sites ont été travaillés par une succession de contrats ou de projets nationaux, régionaux ou départementaux (contrat de développement rural, pôle d'excellence rurale, CUCS...) et qu'ils ont participé à divers réseaux de développement culturel ou d'insertion par la culture, sous l'impulsion en particulier du département qui concentre son accompagnement sur ces territoires. La sensibilité de leurs élus à la culture et à tout projet de décloisonnement explique que certaines communes, généralement les mêmes, s'investissent dans des projets portés par des acteurs extérieurs, institutionnels ou associatifs (Fédération régionale des foyers ruraux...). Cette réalité a permis des habitudes de travail en commun entre les communes les plus investies (autour du conte ou de la lecture, par exemple), ce qui ne les empêche pas de mener par ailleurs leurs propres actions. Mais ces dynamiques fondées sur le volontariat s'inscrivent rarement dans un périmètre administratif donné et cela d'autant moins que ces territoires se caractérisent par une accumulation de périmètres à géométrie variable (intercommunalités, syndicats, pays...).

L'investissement dans l'éducation artistique et culturelle procède au départ de la même logique que celle qui préside à l'investissement dans d'autres projets. Mais l'extériorité de l'impulsion ne constitue pas nécessairement un obstacle à la prise de conscience de la nécessité de mettre en place une politique éducative territoriale. Celle-ci nécessite un accompagnement évident tant des acteurs institutionnels locaux que des structures culturelles qui s'ouvrent progressivement au partenariat avec d'autres et au travail en réseau.

La diversité des configurations locales illustre à quel point l'éducation artistique et culturelle est un miroir des dynamiques locales à l'œuvre et des repositionnements des acteurs en cours aux différents échelons territoriaux. Elle met en lumière le poids des disparités entre départements, selon le degré de leur implication dans le développement de l'éducation artistique et culturelle (variable de l'un à l'autre à l'intérieur d'une même région) et entre communes, selon leurs ressources mais plus encore selon qu'elles hébergent des personnels politiques et des opérateurs culturels en capacité d'élaborer un projet culturel et d'anticiper les réorganisations et les mutualisations à venir. Elle rappelle enfin l'inanité de toute volonté d'uniformisation des démarches, en l'absence de modèle unique pertinent. La stratégie la plus efficace restant celle qui répond le mieux aux enjeux et aux logiques d'acteurs en présence et est capable de mobiliser les compétences et les ressources du territoire concerné.

\section{UN CONTEXTE EN ÉVOLUTION FAVORABLE À LA REDÉFINITION DU SENS DE L'ACTION PUBLIQUE ET DE SES MODALITÉS}

Ce contexte émergent de mise en œuvre de l'éducation artistique interroge les modes d'intervention et d'accompagnement des acteurs régionaux et départementaux. Certes, ceux-ci n'ont jamais été monolithiques ni uniformes et se sont adaptés au fil du temps aux caractéristiques des territoires. Mais tant la diversité des schémas locaux d'organisation de l'éducation artistique et culturelle que l'apparition de nouvelles contraintes qui affectent les sites de manière différentielle, exigent de repenser en partie les démarches et les procédures utilisées, pour les adapter à la diversité des pratiques, des attentes et des ressources des acteurs locaux. Cette réalité invite à réfléchir aux priorités stratégiques de l'action, aux moyens d'améliorer le fonctionnement des «systèmes d'acteurs » et aux méthodologies d'action à promouvoir. 


\section{De nouvelles finalités stratégiques}

Les études de terrain montrent que l'objectif originel de généralisation de l'éducation artistique et culturelle et de réduction des disparités dans l'accès à la culture et à l'éducation artistique et culturelle n'est pas encore atteint mais qu'il demeure, pour l'ensemble des acteurs, un objectif pertinent. Sa réalisation se heurte cependant à la limitation croissante des moyens humains et financiers mis à son service. L'histoire des arts à l'école représente, pour nombre d'acteurs, un levier d'action intéressant pour une diffusion plus large de l'éducation artistique et culturelle, mais il est encore trop tôt aujourd'hui pour mesurer les retombées de ce programme au regard de la généralisation souhaitée. La poursuite de cette finalité rend ainsi plus nécessaire le recours à des structures démultiplicatrices, proches des publics, qui contribuent en retour à renforcer le rôle des collectivités locales. Une nouvelle division du travail est à trouver entre acteurs institutionnels et acteurs culturels, entre structures culturelles et socioculturelles, entre structures instituées et associatives.

Au côté de cet objectif historique apparaît une nouvelle exigence relative à la mise en cohérence des actions, tout au long de la chaîne des acteurs et au plus près des territoires. Le manque de lisibilité de l'offre d'éducation artistique et culturelle, maintes fois souligné, alimente l'impression d'une offre pléthorique, qui peut engendrer une démobilisation des acteurs ou une exacerbation de logiques de concurrence entre ces derniers. Le manque de cohérence des propositions a des origines structurelles : intégration insuffisante, localement, des diverses politiques qui traitent directement ou indirectement d'éducation artistique et culturelle (politiques culturelles, politiques de la jeunesse, politique de la ville, réussite éducative, politique en faveur de la cohésion sociale...), absence de stabilisation (ou complexité) de la répartition des compétences culturelles entre intercommunalités et communes, segmentation historique de la gestion des niveaux scolaires et de la gestion des temps de l'enfant.

Diverses démarches témoignent aujourd'hui du souci d'une meilleure articulation des projets et de la coordination des acteurs. La notion de parcours d'éducation artistique et culturelle permet de relier les différents niveaux scolaires (primaire, collège, lycée), d'articuler les temps de l'enfant et de penser la continuité et la progression des apprentissages dans les propositions faites aux jeunes dans la durée (facilitant ainsi la maîtrise de cette éducation). Des démarches de diagnostic se développent avec la réalisation d'états des lieux destinés à recenser l'offre et les acteurs ou encore les démarches d'évaluation, qui s'intéressent à l'organisation et aux fonctionnements de l'offre. Enfin, l'intérêt pour les démarches de mutualisation tend à se développer, que cellesci visent l'amélioration de la gestion (programmation et communication), des gains de productivité et des économies (désignation d'un seul médiateur pour deux communes, par exemple) ou encore la circulation des savoirs et des savoir-faire, même si les sites étudiés offrent encore peu d'illustrations opérationnelles de telles démarches. Pour autant, il reste encore beaucoup à faire pour favoriser la compréhension, et même la visibilité, des politiques d'éducation artistique et culturelle en cours. L'intégration des différentes propositions émanant des différents échelons territoriaux s'inscrivent encore dans des politiques diverses et mobilisent des acteurs aux profils variés.

\section{L'accompagnement du repositionnement des acteurs}

Devenue un objet de politiques publiques, l'éducation artistique et culturelle se développe aujourd'hui dans un contexte soumis à de nombreuses mutations : poursuite de la décentralisation et ses implications tant politiques que financières pour les collectivités territoriales, réforme territoriale, crispation des finances publiques. Les repositionnements en cours concernent l'ensemble des échelons territoriaux et des acteurs. L'implication croissante des collectivités territoriales dans l'éducation artistique et culturelle, mesurée par l'accroissement des budgets qui lui sont consacrés, modifie la place et le rôle des intervenants tout au long de la chaîne de décision allant du local au national. Dans le même temps, cette implication demeure extrêmement variable d'un territoire à l'autre, avec des communes ou des intercommunalités très investies et, à l'autre extrémité, des communes peu sensibilisées et peu désireuses de s'engager dans l'éducation artistique et culturelle. Cette réalité rend impossible une uniformisation des modes d'intervention et oblige à les adapter à la diversité des contextes locaux.

L'introduction de nouvelles priorités fixées par les collectivités à l'éducation artistique et culturelle, concernant les publics, avec la volonté de s'adresser à d'autres publics que les scolaires ou encore de proposer une offre sur le hors temps scolaire, et concernant la gestion de l'éducation artistique et culturelle, avec l'aspiration à articuler les politiques d'éducation artistique et culturelle et les politiques périphériques (politique de la ville, politiques sociales, politique d'égalité des chances et d'insertion), vient perturber les règles du jeu antérieures tant pour les acteurs institutionnels départementaux et régionaux que pour les acteurs culturels. Du côté de l'Éducation nationale, le développement de politiques territoriales d'éducation artistique et culturelle questionne les modes d'inscription de l'institution scolaire dans ces politiques, dans la mesure où l'établissement scolaire n'est plus la seule porte d'entrée possible dans l'éducation artistique et culturelle, même si elle demeure essentielle aux yeux de tous les acteurs.

L'explosion des besoins d'accompagnement (qu'ils émanent de collectivités encore mal préparées ou de communes très actives en demande d'un dialogue avec l'État) se heurte à la limitation des moyens humains et financiers tant du côté des acteurs institutionnels que des opérateurs culturels, qui ne peuvent être présents partout. Dans ce contexte, il existe un risque d'un repli de la part des institutions régionales et départementales sur leurs seules compétences obligatoires, accompagné d'un désinvestissement ou d'une moindre implication dans des politiques plus transversales. Aussi des décideurs locaux en appellent-ils aujourd'hui à une clarification du rôle de l'État et de ses représentants, et à une clarification de ses engagements auprès des collectivités locales. Des structures culturelles se déclarent dans l'incapacité d'accueillir davantage de publics et d'étendre leur offre, sauf à faire baisser la qualité de leurs propositions. Ces contraintes conduisent à une transformation des stratégies déployées. La première concerne la désignation de territoires prioritaires d'intervention, laquelle soulève de nouvelles questions concernant :

- les critères de sélection de ces territoires, même si l'expérience montre une convergence de vues et de diagnostics entre échelons territoriaux ;

- une définition concertée des territoires prioritaires d'intervention de l'État et de ses partenaires territoriaux, celle-ci n'impliquant pas nécessairement une co-intervention des acteurs centraux, régionaux et départementaux sur ces sites, mais requérant des procédures partagées de répartition des rôles, de suivi 
de comptes rendus et de rapports d'activité, dans une logique de meilleure répartition des moyens disponibles;

- le type de liens à construire avec les territoires désignés comme non prioritaires, afin de savoir ce qu'il s'y passe, de maintenir des possibilités d'échanges et d'apporter des réponses ciblées à leurs demandes.

Une autre stratégie renvoie à la désignation de relais dans les territoires, de manière à démultiplier l'action des acteurs historiques. Mais cette politique, simple en apparence, nécessite un certain nombre de prérequis. Elle suppose que soient préalablement définies les conditions du financement de ces relais et de la pérennité du nouveau fonctionnement mis en place. Elle implique la formation de ces nouveaux médiateurs, l'instauration de lieux de partage et de mutualisation des pratiques, une animation de réseau, afin d'éviter une éducation artistique et culturelle à deux vitesses, susceptible d'ajouter une nouvelle forme d'inégalité aux inégalités territoriales existantes. Cette démarche nécessite des investissements non négligeables à court terme, avant de parvenir à une gestion plus efficace et efficiente à moyen terme. Sous peine de maintenir ce qu'une élue appelle un «plafond de verre » entre les communes qui ont le moyen de professionnaliser à l'échelon local l'éducation artistique et culturelle et celles qui demeurent dans l'incapacité de recruter un médiateur culturel et sont contraintes à des arrangements qui alourdissent l'organisation du fait de leur précarité, sans garantir un niveau de qualité satisfaisant.

La nécessité d'une transformation des procédures et des relations avec les collectivités locales est bien perçue par les conseillers en éducation artistique et culturelle des Drac, en contact avec celles-ci, qui soulignent leurs attentes en matière de modes de négociation et de partenariat. Les cinq configurations repérées et décrites dessinent déjà des pistes d'action différenciées. Ainsi, les exemples de politique intégrée d'éducation artistique et culturelle mettent en lumière la richesse du modèle à l'échelle communale mais aussi l'insuffisance fréquente de liens à l'échelle intercommunale. Dans ce contexte, il devient important de favoriser les initiatives qui s'adressent à des territoires plus larges, débordant le cadre communal. Les approches ciblées et multipolaires posent des problèmes spécifiques d'articulation des différents pôles et de circulation de l'information et des publics entre ceux-ci. Pour les approches segmentées et fondées sur une impulsion extérieure, les leviers d'action sont d'abord politiques et nécessitent un processus de mobilisation et d'accompagnement des acteurs locaux, ce à quoi s'appliquent activement certains départements aujourd'hui. Ils peuvent impliquer la désignation de relais entre les acteurs locaux et les structures culturelles en capacité de piloter et de coordonner l'action de manière pérenne.

Enfin, ce repositionnement en marche des acteurs interroge la place de l'artiste qui, si elle fait consensus, n'en est pas moins fragile, sa légitimité étant souvent questionnée par les institutions. Il interroge celle des structures socioculturelles, dont l'implication dans les partenariats autour de l'éducation artistique et culturelle varie fortement d'une commune à l'autre, en fonction des politiques locales et de l'histoire particulière des liens entre des responsables de structures culturelles et socioculturelles. De même, le rôle des associations culturelles de proximité dans le cadre d'une politique éducative territoriale, souvent plébiscité par des élus locaux en milieu rural tant pour des raisons politiques que budgétaires, fait débat auprès de responsables régionaux et départementaux qui, tout en souhaitant les écarter, hésitent à donner aux collectivités l'impression d'un interventionnisme abusif (surtout lorsque les communes financent de plus en plus l'éducation artistique et culturelle) mais sont soucieux de garantir un niveau de qualité des propositions.

\section{L'évolution des méthodologies d'action}

Le passage d'une politique «territorialisée » d'éducation artistique et culturelle à une politique « territoriale » implique des transformations non seulement organisationnelles et de repositionnement des acteurs, mais aussi de nouvelles méthodologies d'intervention. La généralisation de l'histoire des arts au sein des établissements scolaires modifie la donne en obligeant les enseignants (notamment ceux qui étaient peu sensibilisés jusque-là) et les structures culturelles à mettre en place des projets ; mais elle est porteuse aussi de risques d'une « routinisation » de l'éducation artistique et culturelle.

La diversité des modes d'organisation de l'éducation artistique et culturelle en local rejaillit sur les stratégies de partenariat à mettre en œuvre. La volonté d'une rationalisation des politiques locales d'éducation artistique et culturelle et les restrictions budgétaires conduisent à rechercher de nouveaux modes de faire, comme l'illustrent la référence fréquente aux politiques de mutualisation. Ces quelques constats mettent en lumière l'impossibilité de s'en tenir, au plan opérationnel, à l'incitation des collectivités locales à s'inscrire dans des dispositifs formalisés et l'importance croissante des démarches d'accompagnement qui requièrent d'autres compétences, d'autres savoir-faire.

Mais l'évolution des méthodologies d'action n'est pas seulement défensive, c'est-à-dire destinée à permettre les ajustements nécessaires en vue d'une optimisation des politiques et des ressources. Elle doit tout autant chercher à mieux valoriser et capitaliser les apports des actions innovantes afin de faire avancer l'éducation artistique et culturelle, dans ses pédagogies, dans ses modes de mobilisation des publics éloignés de la culture, dans ses modes de coopération entre enseignants et artistes. On peut déplorer en effet que les innovations réalisées dans ce domaine ne soient pas mieux diffusées, appropriées et qu'elles contribuent insuffisamment à repenser les coopérations entre acteurs et à ensemencer les pédagogies plus classiques dans le domaine des arts et au-delà.

Le développement du numérique est appelé à transformer l'éducation artistique et culturelle. Ses retombées sont d'ores et déjà multiples : il modifie les pratiques culturelles et artistiques, les modes d'information et de communication entre acteurs, les modes de capitalisation de l'information (bases de données, sites, flux RSS, alertes...), les modes de transmission en matière d'éducation artistique et culturelle : la mise en place d'un portail de ressources numériques au service de l'histoire des arts est perçue comme un outil de généralisation de l'éducation artistique et culturelle, dans les zones à l'écart et dotées de peu d'équipements culturels. Il devient urgent aujourd'hui d'explorer les potentiels nés du recours à ces technologies et, en particulier, des formes d'interactivité qu'il rend possible à l'intérieur des projets et entre acteurs.

Si la nouvelle étape du développement de l'éducation artistique et culturelle peut être déstabilisante, en ce qu'elle vient bousculer des logiques d'action et des pratiques qui ont fait la preuve d'une réelle efficacité, elle représente la sanction positive des actions menées au cours des deux dernières décennies et signe le niveau de maturité atteint par des collectivités locales. En dépit des difficultés induites par le contexte de restrictions budgétaires, le rôle accru du local, tout en requérant l'élaboration d'autres formes de gouvernance de l'éducation artistique et culturelle, est porteur d'enjeux stimulants par une mobilisation accrue des ressources humaines, techniques et matérielles des territoires. 


\section{Méthodologie}

Cette étude exploratoire a été mise en œuvre par le DEPS suite à la mise en place d'un groupe de travail sur les questions d'éducation artistique et culturelle et de territoires par le Département de l'éducation, des formations, enseignements et métiers (mission reprise par le Département de l'éducation et du développement artistiques et culturels-DEDAC du Secrétariat général du ministère de la Culture et de la Communication).

L'étude exploratoire sur les modes d'organisation à l'échelle des territoires a porté sur deux régions : le Nord - Pas-de-Calais et les Pays de la Loire. Dans chaque région, trois sites ont été choisis en concertation avec les services déconcentrés du ministère de la Culture et de la Communication. Dans le Nord - Pas-de-Calais, trois sites ont été retenus : les communautés urbaines de Calais et de Valenciennes ainsi que la circonscription scolaire d'Avesnes-sur-Helpe-Fourmies. Dans les Pays de Loire, il s'agit de la ville de Saint-Herblain et des intercommunalités d'Ancenis et de Saumur.

L'étude qualitative a permis de rencontrer au total 144 personnes; dans chaque région, les entretiens ont été conduits auprès d'acteurs institutionnels et culturels (élus et personnels administratifs) intervenant aux différents échelons territoriaux (régional, départemental et local).

\section{RÉSUMÉ}

Le développement de politiques d'éducation artistique et culturelle initiées par les collectivités territoriales depuis une vingtaine d'années donne lieu aujourd'hui à une mosaïque de dispositifs. À partir d'une observation menée en Nord - Pas-de-Calais et dans les Pays de la Loire, et après une présentation de la structuration de la politique d'éducation artistique et culturelle entre État et collectivités territoriales d'un point de vue historique, l'étude analyse les différentes configurations et stratégies développées à l'échelon territorial.

Cinq configurations types sont identifiées : une politique d'éducation artistique et culturelle intégrée à la politique locale, une politique ciblée autour d'une discipline (spectacle vivant par exemple), une gouvernance multipolaire, une approche segmentée où acteurs et dispositifs se juxtaposent et enfin une mise en œuvre externalisée. Enfin, l'étude rappelle que le contexte de mutation politique et technologique est propice à une redéfinition du sens de l'action publique et de ses modalités en faveur notamment du développement des actions d'éducation artistique et culturelle.

\section{ABSTRACT}

The development of artistic and cultural education policies under the aegis of local authorities over the last twenty years has today given rise to a patchwork of systems. Based on a survey conducted in the Nord-Pas-de-Calais and Loire regions, and following a historical overview of the formulation of the artistic and cultural education policy between state and regional authorities, this study analyses the various configurations and strategies developed at regional level.

Five typical configurations have been identified: a policy of cultural and artistic education integrated with national policy, a policy based around a particular discipline (live entertainment, for example), multi-disciplinary governance, a segmented approach in which the various bodies or systems work alongside each other, and finally external implementation. Ultimately, the study reminds us that the context in which political and technological change takes place shapes a redefinition of the meaning of public action in all its forms to encourage the development of artistic and cultural education initiatives.

Tous les documents publiés par le DEPS sont téléchargeables sur http://www.culture.gouv.fr/deps

Le DEPS n'assurant pas de diffusion physique de ces documents, nous vous proposons de vous informer régulièrement des parutions par message électronique. Pour ce faire, merci de bien vouloir nous communiquer votre courriel à l'adresse contact.deps@culture.gouv.fr en indiquant comme sujet du message : « diffusion des collections du Deps ». 